\title{
Die Präparation der weißen Substanz des Gehirns bei Stensen, Willis und Vieussens*
}

Von Adolf Faller

\section{Stensen - Willis - Vieussens}

Was veranlaßt uns, die Namen dieser drei bedeutenden Makroskopiker der Neuroanatomie des 17. Jahrhunderts in dieser Reihenfolge miteinander zu verknüpfen? Willis ist der älteste der drei (1621-1675). Ihm folgt Stensen (1638-1686). Raymond de Vieussens ist der jüngste (1661-1716). Das neuroanatomische Hauptwerk von Willis ist die «Cerebri Anatome», die 1664 in London erschien. Hinsichtlich der weißen Substanz des Gehirns interessiert uns mehr sein vorwiegend klinisches Werk «De Anima Brutorum» von 1672. Stensen hielt seinen «Discours sur l'anatomie du cerveau» $1665 \mathrm{im}$ Kreise von Thévenot in dessen Landhaus in Issy bei Paris. Darin beschäftigt er sich eingehend mit der «Cerebri Anatome» des Willis, die im Vorjahr erschienen war. Gedruckt wurde der «Discours» allerdings erst 1669 unter dem Patronat von Thévenot durch Robert de Ninville. Stensen, der schon 1665 Paris verlassen hatte, konnte sich nicht mehr darum kümmern. So kam es, daß die Abbildungen ohne Legenden geblieben sind und daß die beiden Abbildungen der Tafel 4 ohne vereinfachende Strichzeichnung gedruckt wurden. Die «Neurographia Universalis» von Vieussens erschien 1684 in Lyon. Weder Willis noch Vieussens nehmen auf Stensens «Discours» Bezug. Wenn es auch möglich ist, daß ihnen der französische Originaltext nicht zur Verfügung stand - betrug die damalige Auflage ja höchstens ein paar hundert Exemplare -, so dürfte ihnen die lateinische Übersetzung durch den humanistischen Arzt Guido Fanoisius, Leiden 1671, bekannt gewesen sein: «Nicolai Stenonis de Cerebri Anatome Dissertatio, spectatissimis viris DD societatis apud Dominum Thévenot Parisiis collecta, dicata atque a Gallico exemplari Parisiis edita An. 1669 Latinitate donata, Opera et Studio Guidonis Fanoisii LL. AA. M. ( = liberalium artium Magister) et Med. Doctor. Lugd. Batav. Apud Felicem Lopez, Anno 1671. Vieussens erwähnt auch nicht den von ihm so hoch geachteten Willis. Es bleiben nur die

* Prof. Heinrich Buess zum 70. Geburtstag gewidmet von seinem einstigen Klassenkameraden am Humanistischen Gymnasium Basel. 
Jahrzahlen 1669, 1672 und 1684, die wohl die entsprechenden Prioritäten festlegen, aber keine direkten Abhängigkeiten nachweisen lassen. Stensens «Discours» ist eine Programmrede zur Hirnforschung. Er selber hat vor allem durch den Mediosagittalschnitt und die «Orthographia transversa triplex» (C. Thomae Filius Bartholin, 1679) zur Hirnpräparation beigetragen. Er gab der Hoffnung Ausdruck, daß es eines Tages möglich sein werde, den Faserzügen der weißen Substanz zu folgen. Die ersten Ansätze zu einer

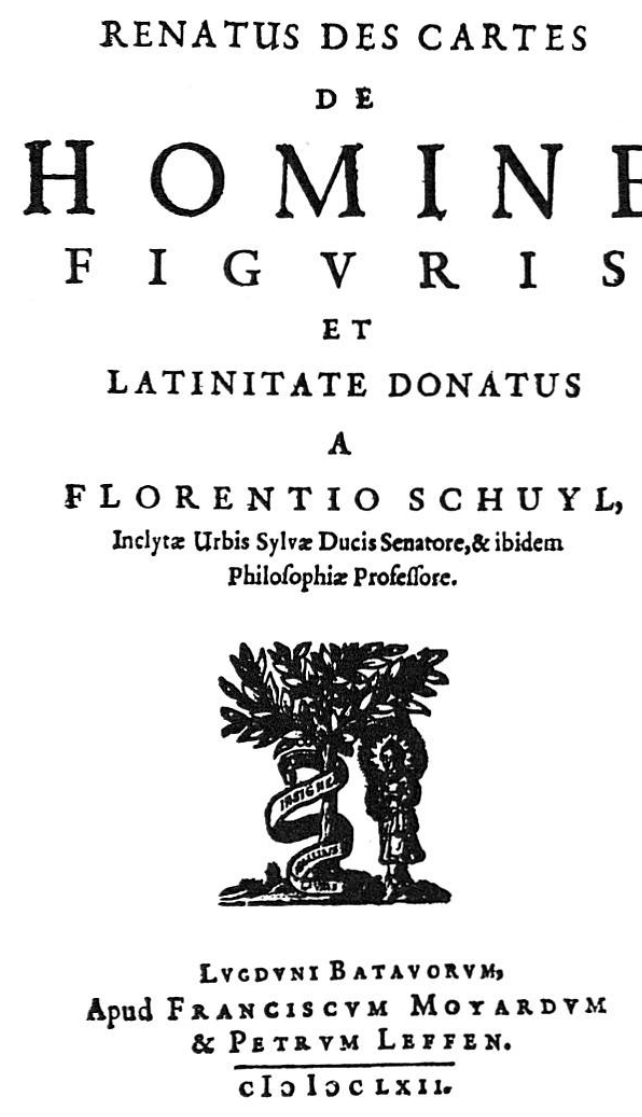

Abb. 1

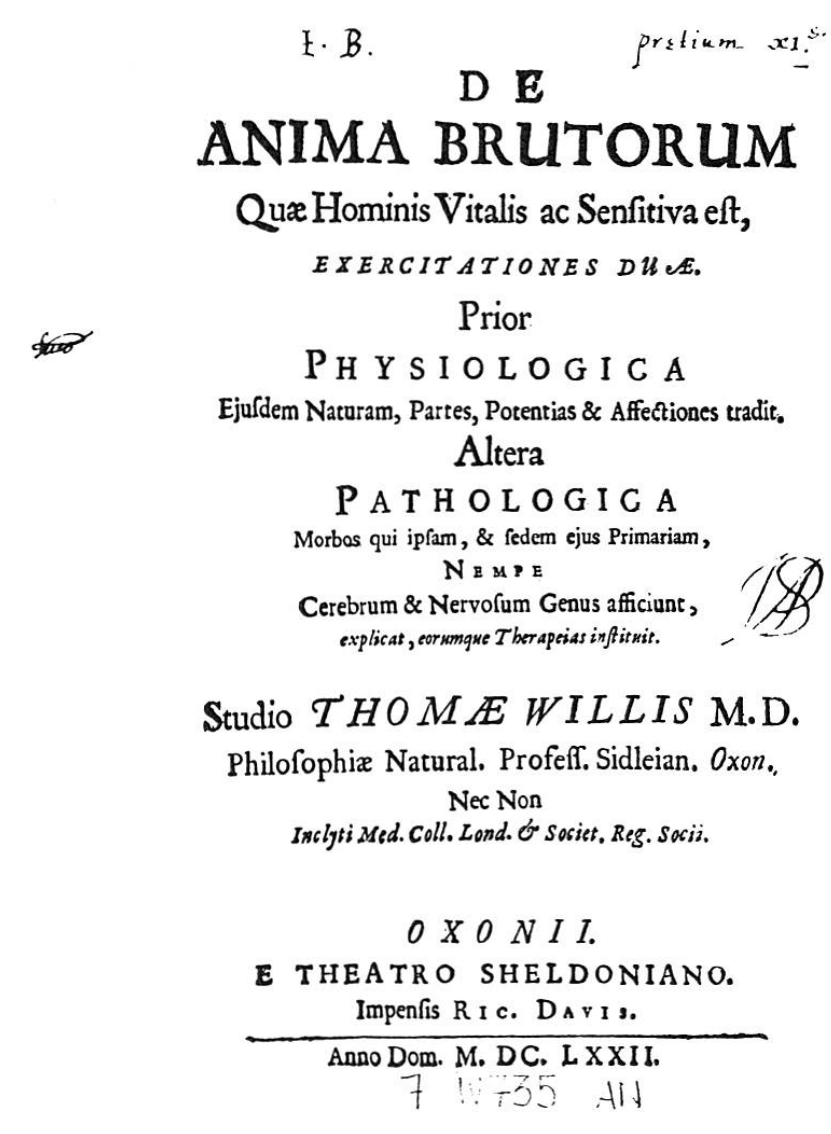

Abb. 2

Abb.1. Titelblatt der lateinischen Ausgabe von Descartes' Anatomie durch Florentius Schuyl (1662). Medizingeschichtliche Bibliothek Fribourg. Auf diese Ausgabe bezieht sich Stensen in seinem Brief vom 26. August 1662 an seinen einstigen Lehrer Thomas Bartholin

Abb.2. Titelblatt von Willis' «De Anima Brutorum». Erstausgabe Oxford 1672. Medizinhistorisches Institut, Universität Zürich. Das Werk strebt eine Synthese von Anatomie, Physiologie, Pathologie und Theologie an. Es erschien 3 Jahre nach Stensens Pariser «Discours» (gehalten 1665, gedruckt 1669), nimmt aber keinerlei Bezug darauf 
solchen Präparation unter Abschaben der grauen Substanz mit entsprechenden Abbildungen haben erst Willis und Vieussens gegeben. In diesem Sinne rechtfertigt sich die Reihenfolge Stensen - Willis - Vieussens, wie wir in den folgenden Kapiteln zeigen wollen. Wir geben von jedem der drei Untersucher die wichtigsten Lebensdaten und den Bildungsgang, analysieren kurz das einschlägige Hauptwerk und zitieren, was uns für die Präparation der weißen Substanz des Gehirns wichtig erscheint.

\section{Nicolaus Stenonis}

\section{Leben und Bildungsgang (M.Bierbaum und A.Faller, 1979)}

Niels Stensen, wie er in seiner dänischen Heimat heißt, wurde 1636 in Kopenhagen als Sohn eines Goldschmiedes geboren. Er besuchte dort die protestantische Liebfrauenschule und die Universität, wo der berühmte Thomas Bartholin sein Studienberater war. Aufzeichnungen zwischen dem 8. März und dem 3. Juli 1659, bekannt als Chaos-Manuskript, geben einen guten Einblick in die wissenschaftlichen und religiösen Fragen, die den jungen Studenten beschäftigten. Stensen studierte nur deshalb Medizin, weil das in der damaligen Zeit die einzige Möglichkeit war, biologische Studien zu treiben. Die Wirren des Schwedenkriegs ließen ihn seine Ausbildung zwischen 1660 und 1664 in Amsterdam und Leiden fortführen. 1660 entdeckte er den nach ihm benannten Ausführgang der Ohrspeicheldrüse. Der daran sich knüpfende Prioritätsstreit mit dem Anatomen Gerhard Blaes führte zum Wechsel von Amsterdam nach Leiden, wo er besonders von dem Iatrochemiker de le Bö̈ (Sylvius) beeinflußt wurde. Er bearbeitete vor allem die Anatomie der Drüsen und der Muskeln und sezierte häufig das Gehirn nach der Methode des Sylvius. Als Stensen nach dem Tod seines Stiefvaters 1664 nach Kopenhagen zurückkehren mußte, verlieh ihm die Universität Leiden «in absentia» den Doktortitel der Medizin. Nach Erhalt seines Erbteils zog er nach Paris und blieb dort über zehn Monate im Hause seines gelehrten Gönners Melchisedech Thévenot. Dort hielt er 1665 seinen berühmten «Discours sur l'anatomie du cerveau», den Herrlinger (1966) zu den großen «Ignoramus»-Bekenntnissen der Weltliteratur rechnet. Eigentlich handelt es sich um ein Programm künftiger Hirnforschung. Indem er Descartes' Irrtum über Lage und Funktion der Zirbeldrüse richtigstellte, leitete er die Kritik der cartesianischen Philosophie ein. Von Paris reiste Stensen nach Montpellier, wo er mit bedeutenden Vertretern englischer 
Wissenschaft und der neugegründeten Royal Society in Berührung kam. Dann aber zog es den kaum 28jährigen an den Hof des Großherzogs der Toscana. In der «Accademia del Cimento» fand er das ihm zusagende wissenschaftliche Klima und großzügige Unterstützung seiner Arbeiten. Hier entstand das «Myologiae Specimen» mit seinen geometrischen Modellvorstellungen. Ihm folgte die Arbeit «Canis Carchariae dissectum Caput», worin er die Bedeutung der Fossilien für die Geologie erwog. Dann schloß er das Manuskript für den «Prodromus», einer kurzen Zusammenfassung der Geologie der toskanischen Landschaft, ab. Die Jahre 1666 und 1667 führten zum Übertritt vom lutherischen Glauben zur katholischen Kirche. 1668 trat er seine zweijährige große Reise durch Südost-Europa an, um Material für weitere mineralogische Untersuchungen zu sammeln. Der zweite Aufenthalt in Florenz endigte mit seiner Rückkehr nach Kopenhagen als «Anatomicus Regius», veranlaßt durch einen Ruf von König Christian V. Aus seiner akademischen Antrittsvorlesung stammt das berühmte Wort «Pulchra, quae videntur, pulchriora, quae sciuntur, longe pulcherrima, quae ignorantur» - «Schön ist, was wir sehen, schöner was wir durch die Vernunft erkennen, aber das Allerschönste bleibt uns verborgen». Immer mehr fand Stensen diese letzte und höchste Stufe der Erkenntnis in der Welt des Glaubens. 1675 nahm er das Amt eines der Prinzenerzieher am toskanischen Hof an. Eifrig betriebene theologische Studien führten ihn zum Priestertum. 1677 wurde er zum Bischof von Titiopolis geweiht. Von 1677 bis 1680 weilte er als Apostolischer Vikar am Hofe von Hannover, wo er mit Leibniz in Kontakt kam. Beide hatten manche gemeinsame Züge, aber eine zu verschiedene Einstellung, für den einen war der Glaube, für den andern die Vernunft die Richtschnur des Handelns. Nach dem plötzlichen Tod des katholischen Herzogs übernahm sein protestantischer Bruder die Regierung. Stensen mußte Hannover verlassen. Der Fürstbischof von Paderborn Ferdinand von Fürstenberg erbat sich den Apostolischen Vikar als Weihbischof für Münster. Von 1680 bis 83 wirkte Stensen im Bistum Münster in der Seelsorge. Er firmte in über 200 Pfarreien und stärkte die Ordnung in den klösterlichen Gemeinschaften von Ringe und Rosental. Stensen hatte bald eingesehen, daß die meisten Mißstände im Bistum vom Ungenügen der Pfarrer abhingen. Er gab deshalb die Schrift «Parochorum hoc age» «Hirtenpflicht» in Florenz in Druck, erreichte aber damit die Pfarrherren des Bistums Münster nicht mehr. Die Weihe der Neu-Priester brachte ihn in schwere Gewissenskonflikte: die Interessen der kirchlichen Reform und die Interessen des Domkapitels widersprachen sich häufig. Zum offenen Kon- 
flikt zwischen Stensen und dem Domkapitel kam es bei der Wahl des neuen Münsterer Bischofs. Stensen protestierte in Rom gegen die Verwendung von Geldmitteln bei der Wahl. Am Wahltag selber verließ er Münster und wandte sich nach Hamburg, dem Zentrum der nordischen Mission. Er fand ein Unterkommen bei dem Arzt Theodor Kerckring, dem einstigen Studienkameraden und Vertreter des toskanischen Großherzogs. Die Streitigkeiten unter den Katholiken verunmöglichten jede gedeihliche Wirksamkeit. Da tat sich ihm ein neues Feld missionarischer Tätigkeit in Schwerin auf. Als einfacher Priester wirkte er in der kleinen Gemeinde durch die Aszese seines Lebens und durch seine Wohltätigkeit. 1686 starb er in Schwerin an einem durch ein Steinleiden verursachten Ileus. Katholiken und Lutheraner betrauerten ihn gleichermaßen. Die Beisetzung erfolgte zunächst im protestantischen Dom von Schwerin bis zum endgültigen Begräbnis in der Krypta von San Lorenzo in Florenz. Bischof Stensen war vor allem als Pastoraltheologe im Sinne der individuellen Seelsorge sowie als Kontroverstheologe im Geiste moderner Ökumene bedeutend: seiner von $\mathrm{Haß}$ zerrissenen Zeit hat er vorgelebt, daß nur Versöhnung, Dienst und Liebe die Menschen erneut zusammenführen können.

\section{Der «Discours sur l'anatomie du cerveau» von 1665}

Dieser Rede zur Hirnforschung gingen zahlreiche Hirnsektionen nach der Methode des Franz de le Boë voraus. Einen Hinweis finden wir schon in der Amsterdamer Zeit bei der Entdeckung des Stensen-Ganges. Die Herausgabe von Descartes" «De Homine» 1662 durch den Philosophieprofessor Florentius Schuyl (Abb. 1) lenkte Stensens Interesse auf das Gehirn. So begeistert Stensen von Descartes' Methodik ist, so kritisch prüft er ihre anatomischen Einzelheiten. Der «Discours» läßt vier Hauptabschnitte erkennen: das Eingeständnis unserer Unwissenheit vom Bau des Gehirns, die Aufzählung der wichtigsten Irrtümer der zeitgenössischen Hirnanatomie, die Notwendigkeit neuer Forschungsmethoden und der Entwurf eines umfassenden Forschungsprogramms für die Hirnanatomie der Zukunft. Wenn wir von Malpighis «Epistola de cerebro» (Bologna 1664) absehen, so ist die Hirnanatomie dieser Zeit rein makroskopisch. Stensen hat dazu einen wesentlichen Beitrag geleistet. Er hat die Hirnsektion nach Franz de le Boë zum Mediosagittalschnitt, wie wir ihn noch heute üben, weiterentwickelt. Ferner führte er makroskopische «Serien»-Schnitte ein («Orthographia tranversa triplex»). Nachdrücklich betonte er den großen Wert vergleichend-anatomi- 
scher Hirnuntersuchungen. Der innere Bau der grauen und weißen Substanz des Gehirns blieb weiterhin unbekannt. Das Neue bei Stensen ist, daß er auf die Notwendigkeit einer Erforschung der innern Struktur hingewiesen hat, die ja bereits Descartes (1662) vermutet hatte, sagt er doch auf Seite 72 in «De Homine»: «Totum enim cerebrum nihil aliud est, quam textus quispiam peculiari modo concinnatus»- «Denn das ganze Gehirn ist nichts anderes als auf besondere Art zusammengefügtes Gewebe». Von einer Analyse der weißen Faserung versprach sich Stensen wichtige Einsichten in die Bahnenlehre. Auch hier hat er ein Feld der Forschung abgesteckt, das er andern zur Bearbeitung überließ.

\section{Stensen und die Präparation der weißen Substanz}

Es lohnt sich, die Stellen, welche im «Discours» von der weißen Substanz handeln, zusammenzustellen. Man erhält so eine Vorstellung von dem, was sich Stensen bei dem Begriff weißer Substanz gedacht hat. Es ist mengenmäBig wenig, aber dieses Wenige ist zuverlässig und entspricht ganz seiner kritischen Arbeitsweise. Schon auf Seite 3 seines «Discours» kommt das zum Ausdruck: «... je ne laisse pas d'estre tres-persuadé que ceux qui cherchent une science solide, ne trouveront rien qui les puisse satisfaire, dans tout ce que l'on a écrit du cerveau» - «noch immer bin ich völlig davon überzeugt, daß diejenigen, welche eine solide Wissenschaft suchen, in allem, was vom Gehirn geschrieben wurde, nichts Befriedigendes finden werden». Etwas weiter auf der gleichen Seite schreibt er: «Il ne faut que voir dissequer la grande masse de matiere, qui compose le cerveau, pour avoir sujet de se plaindre de cette ignorance. Vous voyez sur la surface, des diversitez qui méritent l'admiration; mais quand vous venez à pénetrer jusqu'au-dedans, vous n'y voyez goutte; tout ce que vous en pouvez dire, c'est qu'il y a deux substances differentes, l'une griseatre $\&$ l'autre blanche; que la blanche est continuë aux nerfs qui se distribuent par tout le corps»- «Man muß nur bei der Dissektion der großen Masse, die das Gehirn bildet, zusehen, um allen Grund zu haben, diese Unwissenheit zu beklagen. An der Oberfläche sieht man allerlei, was unsere Aufmerksamkeit verdient. Aber wenn man ins Innere eindringt, so sieht man gar nichts. Alles, was sich sagen läßt, ist, daß es zwei verschiedene Substanzen gibt, die eine grau, die andere weiß und daß die weiße in die Nerven übergeht, die sich im ganzen Körper verteilen». Auf Seite 4 fährt Stensen weiter: «Si on nous demande, Messieurs, ce que c'est ces substances, de quelle maniere les nerfs se ioignent dans la substance blanche, 
jusques où les extremitez y avancent, c'est là où l'on doit avouër son ignorance»...- «Wenn man sich fragt, ihr Herren, was diese Substanzen sind, auf welche Art die Nerven sich mit der weißen Substanz verbinden, wie weit die Nerven eindringen, dann muß man seine Unwissenheit eingestehen.» Stensen bedauert es, so wenig Sicheres über den innern Bau des Gehirns zu wissen. An der Oberfläche hat er offensichtlich die Gyri und Sulci gesehen, ohne indessen hier näher darauf einzutreten. Die Hirnnerven hält er für Fortsätze der weißen Substanz. Klar und deutlich gesteht er ein, daß er vom innern Bau der grauen und weißen Substanz nichts wisse und die Verbindungen zwischen Hirn und Hirnnerven nicht kenne. Mit den damaligen Methoden konnte man nicht feststellen, daß die Nervenfasern entweder in grauen Kernen entspringen oder an solchen endigen. Die Unterscheidung von grauer und weißer Substanz geht auf die «Anatomicae Praelectiones» (Rom 1586) des Archangelo Piccolomini (1526-1586) zurück, der von Sprengel zu den mittelmäßigen Anatomen des 16. Jahrhunderts gerechnet wird. Als «Cerebrum» stellt er die Gesamtheit der grauen Substanz der weißen, die er «Medulla» nennt, gegenüber. Von der Struktur der weißen Substanz sagt Stensen auf Seite 4 seines «Discours»: «Car de dire que la substance blanche n'est qu'un corps uniforme, comme seroit de la cire, où il n'y a point d'artifice caché, ce seroit avoir un sentiment trop bas, du plus beau chef-d'œuvre de la nature. Nous sommes assurez, que par tout où il y a des fibres dans le corps, par tout elles observent une certaine conduite entr'elles, plus ou moins composée, selon les opérations ausquelles elles sont destinées. Si la substance est par tout fibreuse, comme en effet elle le paroist en plusieurs endroits il faut que vous m'avouiez que la disposition de ces fibres, doit estre rangée avec un grand art, puisque toute la diversité de nos sentimens, \& de nos mouvemens en dépend. Nous admirons l'artifice des fibres dans chaque muscle; combien les devons nous admirer davantage dans le cerveau, où ces fibres renfermées dans un si petit espace, font chacune leur operation, sans confusion \& sans desordre» - «Denn zu sagen, die weiße Substanz sei ein gleichförmiger Körper etwa wie Wachs, wo es keine verborgene Struktur gebe, wäre eine zu einfache Vorstellung vom größten Kunstwerk der Natur. Wir wissen, daß es überall, wo es im Körper Fasern gibt, diese eine bestimmte komplizierte Anordnung zeigen, je nach der Funktion, für welche sie bestimmt sind. Wenn die (Hirn) Substanz überall faserig ist, wie dies an mehreren Orten der Fall ist, so müssen Sie mir zugestehen, daß sie sehr kunstvoll angeordnet sein muß, weil die unterschiedlichen Empfindungen und unsere Bewegungen davon abhängen. Wir bewundern die kunstvolle 
Anordnung der Fasern im Muskel, um wieviel mehr müssen wir sie im Gehirn bewundern, wo die Fasern auf so kleinem Raum ihre Funktion ohne Verwirrung und Unordnung ausüben.» Ausgehend von der Tatsache, daß faserige Strukturen im Körper weit verbreitet sind, lehnt Stensen es ab, die weiße Substanz als homogen zu betrachten. In seiner gewohnten Vorsicht betont er aber, daß er Faserstrukturen nur an einzelnen Orten deutlich gesehen habe. Hirnschnitte können das nicht zeigen. Auch die Entfaltung der Windungen ist dazu kaum geeigneter. Auf Seite 7 betont er, daß allein die Trennung von grauer und weißer Substanz weiterführen kann: «une séparation du corps gris, d'avec la substance blanche, passe un peu plus outre». Schließlich entwickelt er auf Seite 8 die geniale Idee der Faserpräparation: «Pour moy, ie tiens que la vraye dissection seroit, de continuer les filets des nerfs au travers de la substance du cerveau, pour voir par où ils passent, \& où ils aboutissent. Il est vray que cette maniere est pleine de tant de difficultez, que ie ne sçay, si on oseroit iamais esperer d'en venir à bout, sans des preparations bien particulieres. La substance en est si molle, \& les fibres si delicates, qu'on ne les sçauroit à peine toucher, sans les rompre»- «Ich selber halte dafür, daß das richtige Vorgehen wäre, die Faser(Bündel) durch die Hirnsubstanz zu verfolgen, um festzustellen, wo sie durchziehen und wo sie enden. Es stimmt, daß diese Art der Präparation so schwierig ist, daß ich nicht weiß, ob man das ohne besondere Präparationsmethoden erreichen kann. Die (Hirn) Substanz ist so weich und die Faser (Bündel) so verletzlich, daß man kaum sich getraut, sie anzurühren, ohne sie zu zerreißen.» Der letzte Satz läßt vermuten, daß Stensen Versuche in dieser Richtung gemacht hat, daß sie ihn aber nicht befriedigt haben. Es brauchte noch fast dreihundert Jahre, bis mit Hilfe der Formolfixierung, kombiniert mit dem Durchfrieren der Gehirne (Ludwig und Klingler 1938 und 1956), wirklich schöne Faserpräparate möglich wurden. W as Stensen «fibres» nennt, waren wohl Faserbündel, wie sie sich mit der Pinzette fassen lassen. Einzelne Nervenfasern wurden erst 1781 von Fontana erkannt. Die Idee einer Faserpräparation der weißen Substanz ist viel bewundert worden. Sie wird von Clarke und O'Malley (1968) auf Seite 584 ihres Lesebuchs zur Geschichte des Zentralnervensystems besonders hervorgehoben: "Steno has suggested moreover, that one way of studying white matter was "to follow the nerve filaments through the substance of the brain to find out where they go and where they end'. He gave no account of dissections based on this suggestion, but Willis, in his 'De anima brutorum' (Oxford, 1672, Cap. IV), published three years later, mentioned his experience with this methode" - «Stensen 
schlug außerdem vor, daß ein Weg zur Erforschung der weißen Substanz wäre, «die Nervenfasern durch die Hirnsubstanz zu verfolgen, um herauszufinden, wie sie verlaufen und wo sie enden>. Er gibt aber keine Forschungsresultate aufgrund dieser Methode. Hingegen berichtet Willis in seinem Buch 〈De anima brutorum〉 (Oxford 1672, Cap. IV) drei Jahre später über seine Erfahrung mit dieser Methode.» Stensen hat sich besonders für den Streifenkörper (Corpus striatum) interessiert mit seinem Wechsel von Grau und Weiß. Auf Seite 11/12 sagt er: «... si vous faites une séparation du corps gris, d'avec la substance blanche, vous verrez que ces rayes, ne sont toutes que d'une mesme nature; c'est à dire qu'elles font partie de la substance blanche du corps calleux, qui va vers la mouëlle du dos, separée en diverses lamelles, par l'entremise de la substance grisâtre» - «... wenn man graue und weiße Substanz auseinandertrennt, so sieht man, daß diese Streifen alle von gleicher Natur sind, d. h. weiße Substanz des Corpus callosum, die von grauer Substanz in Lamellen unterteilt zum Rückenmark zieht.» Er kritisiert Willis, der in der Capsula interna auf- und absteigende Fasern annimmt. Willis hat das Richtige geahnt, aber beweisen ließ sich das damals nicht. Insofern ist Stensens Kritik berechtigt. Der Begriff Corpus callosum wurde damals bedeutend weiter gefaßt als heute. Man verstand darunter alle weiße Substanz des Hirnstamms. Auf Seite 31 des «Discours» schreibt Stensen: «Le corps Calleux, selon l'usage commun, signifie la substance blanche du Cerveau, qu'on void quand on en sépare les deux parties latérales; mais il est vray que cette partie est entierement semblable au reste de la substance blanche du Cerveau» - «Als Balken bezeichnet man gewöhnlich die weiße Substanz des Gehirns, welche man zu sehen bekommt, wenn man beide Seitenteile voneinander trennt. Es stimmt auch, daß diese Partie völlig der restlichen weißen Substanz gleicht.» Stensen hat auch bereits Unterschiede im Bau der weißen Substanz bei verschiedenen Tieren im Vergleich zum Menschen gekannt: «Il y a des animaux, où les fibres se voyent plus aisément que dans l'homme» - «bei manchen Tieren sind die Faser (Bündel) leichter zu sehen als beim Menschen» (Seite 56 des «Discours»). Es stimmt dies mit den Angaben überein, die Stensen im Brief an Thomas Bartholin vom 26. August 1662 macht, daß er viele Tiergehirne seziert hat. Ergebnisse von Faserpräparationen der weißen Substanz, wie er im «Discours» vorschlägt, erwähnt er jedoch nirgends. Bedeutende Hirnanatomen wie Burdach (1819), Foster (1901) sowie Clarke und O'Malley (1968) haben ihrem Bedauern Ausdruck gegeben, daß Stensen keine Zeit gefunden hat, das von ihm im «Discours» aufgestellte Forschungsprogramm zu verwirklichen. Immerhin zeigt die 
Zusammenstellung der verschiedenen Urteile über den «Discours» im Verlaufe von drei Jahrhunderten (Faller, 1981), daß sein Einfluß bedeutend gewesen sein muß.

\section{Thomas Willis}

Leben und Bildungsgang (H. R. Isler, 1965)

1621 wurde Thomas Willis in Great Bedwin (Wiltshire) geboren. Er besuchte zunächst eine Privatschule in Oxford und trat in die Dienste von Canonicus Iles am Christ Church College. Wie es der scholastische Lehrgang vorschrieb, studierte Willis zunächst die «Artes liberales» und erst anschließend Medizin. Seiner Ausbildung nach ist er Aristoteles, Galen und den Scholastikern verpflichtet. Methodisch jedoch folgte er der «neuen Naturwissenschaft»: Harvey, Paracelsus, den Iatrochemikern und der Schule von Gassendi. Nach Ausbruch des englischen Bürgerkrieges 1642 residierte König Charles I zunächst in Oxford. Willis befand sich unter den angeworbenen Freiwilligen. Er promovierte im gleichen Jahr zum «Magister artium». 1646 ergab sich die Festung Oxford den puritanischen Rebellen. Der anglikanische Gottesdienst wurde verboten. Dr. Iles, der Dienstherr von Willis, wurde abgesetzt. Mitten in diesem Umsturz promovierte Willis zum «Baccalaureus medicinae» und erhielt die Praxislizenz als Arzt. Trotzdem der hochkirchliche Gottesdienst in Hause von Willis heimlich weitergeführt wurde, stand sich dieser mit der Kommission der Neuerer nicht schlecht. Sie empfahl ihn zur Aufnahme in die Colleges. Die Puritaner zogen bedeutende Vertreter der neuen experimentellen Richtung nach Oxford. Diese bildeten die Gruppe des «Invisible College» und beriefen sich auf das «Novum Organon» des Bacon. Sich selber nannten sie die «Vertuosi» und bekämpften die unumschränkte Autorität des Aristoteles. Zu diesem Kreis gehörten auch Christopher Wren, Architekt der neuen St. Pauls Kathedrale, und William Lower, der später seinem Lehrer nach London folgte. 1660 wurde das Regiment der Puritaner gestürzt und König Charles II kam an die Regierung. Wohl durch den Einfluß des spätern Erzbischofs von Canterbury, Gilbert Sheldon, wurde Willis «Sedleys Professor» auf dem Lehrstuhl der Naturphilosophie in Oxford. Nachdem der Erzbischof ihn nach London berufen hatte, behielt Willis nominell den Oxforder Lehrstuhl auch weiterhin bei. Diese seine Stellung als Universitätsprofessor und die Mitgliedschaft bei der eben gegründeten «Royal Society» verschafften ihm internationales Ansehen. 
Zwischen 1664 und 1672 erschienen seine drei großen Werke über das Zentralnervensystem und dessen Erkrankungen die «Cerebri Anatome», «Pathologiae Cerebri et Nervosi Generis Specimen» und «De Anima Brutorum». Von der «Pharmaceutica Rationalis» erschien zu Willis Lebzeiten nur der erste Teil. Er starb im November 1675. Ein Jahr nach seinem Tode erschienen in Genf und Lyon seine «Opera omnia».

\section{Die «Cerebri Anatome» von 1664 und «De Anima Brutorum» von 1672}

Als Anatom hatte Willis besonders das Zentralnervensystem untersucht, dem auch vor allem seine klinischen Interessen galten. Die traditionelle Lehre von den «Spiritus» hat Willis chemisch zu unterbauen versucht. Naturgemäß war die experimentelle Basis der damaligen Zeit zu schmal dafür. Aber seine anatomischen und klinischen Untersuchungen erhielten dadurch einen bisher unbekannten dynamisch-energetischen Charakter, welcher der Neurologie und Psychiatrie besser gerecht wurde. Auffallend ist, wie oft Willis in seinen gewagten Hypothesen moderne Vorstellungen vorweggenommen hat. Die Grundlage aber blieb für ihn stets die klinische Beobachtung. Sein Hauptanliegen war, moderne Naturwissenschaften und Theologie in ein umfassendes Gesamtsystem zu bringen. Willis verbindet in sich scholastische Tradition und die neuen iatrochemischen Vorstellungen. $\mathrm{Daß}$ seiner Synthese große Mängel anhaften, wird kaum verwundern. Manche seiner Ideen sind nur im Rahmen seines ganzen Lebenswerkes zu verstehen. Daher kommt es auch, daß Willis in vielem außerordentlich widersprechend beurteilt worden ist. Die einen sahen in ihm einen bahnbrechenden Neuerer der experimentell naturwissenschaftlich begründeten Medizin, einen Schöpfer neuer Begriffe und neuer Vorstellungen. Andere beurteilten ihn als einen spitzfindigen Scholastiker, einen Wirrkopf und Phantasten. All das zusammen prägte Willis. Dazu kam noch, daß er in einem komplizierten, schwer verständlichen Latein schrieb. So ist es gekommen, daß er und sein Werk außerordentlich widersprechend beurteilt worden sind (Isler 1965). Seine Vorstellung von der Tierseele wendete sich gegen Descartes, der im Tier nur eine mechanische Maschine erblicken wollte. Auch muß Willis Descartes gegenüber als der eigentliche Schöpfer der Lehre von den Reflexen gelten. Ferner ist er der Begründer der Lehre von den unwillkürlichen Funktionen des autonomen Nervensystems und einer umfassenden Psychopathologie. 


\section{Willis und die Präparation der weißen Substanz}

Willis schreibt in «De Anima Brutorum» (Abb. 2), daß die Tierseele der vitalsensitiven Seele des Menschen entspricht. Sie ist körperlich und erlischt mit dem Körperleben. Außer ihr besitzt der Mensch aber noch eine unsterbliche rationale Seele. Für ihn ist der Aufbau des Gehirns aus grauer und weißer Substanz eine Selbstverständlichkeit. Auf Seite 22 im 4. Kapitel ${ }^{1}$ bemerkt er: «Uterque $\dot{\varepsilon} \gamma x \dot{\varepsilon} \varphi \alpha \lambda o v$ duplici constat substantia, scilicet corticali, quae ut plurimum pro spirituum receptione, \& medullari, quae pro illorum dispensatione, \& exercitio inservit» - «Beide Gehirne (Großhirn und Kleinhirn) bestehen aus zweierlei Substanz, aus Rindensubstanz, die vor allem der Aufnahme der «Spiritus〉 dient, und Marksubstanz, die deren Verteilung und Disposition zu dienen hat.» Die weißen Faserbündel sieht er als eine Art innerer Nerven an, «nervi intrinseci». Auf Seite 24 spricht er von der neuen Präparationstechnik: «At vero quoniam objicitur, me hos non omnes, \& forsan haud satis exacte, descripsisse; idcirco ut ductus isti medullares melius conspicerentur, nuper aliam \& paulo accuratiorem cerebri Anatomen instituimus; nempe partes ejus cultelli cuspide leniter scalpendo, substantiam molliorem \& subfuscam cerebri cortici affinem, ubique amovimus, albidiori \& duriori relicta; quo pacto in variis cerebri, \& medullae oblongatae locis, plures chordae medullares, quasi distincti nervi, inter se invicem, cumque aliis corporibus albidis sive medullaribus, mire communicantes, in conspectum prodibunt. Quandoquidem igitur haec administratio Anatomica spirituum ductus secretiores, motusque ad regiminis animalis arcana spectantes, valde conspicuos reddit, libet hic novam cerebri evoluti, \& praecipue in locis quasi excarnati, figuram unam aut alteram exhibere ... Itaque in cerebro exempto, \& juxta methodum nostram evoluto, sectio inter prominentias orbiculares, scilicet inter testes \& nates ita instituatur, ut cum illae integrae, \& glandulae pinealis in medio divisae partes ab invicem seponuntur, cavitas utrisque substrata pateat (prout in Tab.6 Fig. 1 AbE AbcCDT). Tunc facile apparebit prominentias dictas testes medullae oblongatae epiphyses medullosas esse, quae cerebelli caudicibus cohaerentes, inde versus cerebrum spectant, atque inter hoc \& illud commercia sustinere videntur» - «Aber da man mir den Vorwurf machen könnte, ich hätte nicht alle (Markbündel) und diese auch nicht genügend genau beschrieben, habe

1 Meine Seitenangaben entsprechen dem unbebilderten Exemplar «De Anima Brutorum», London 1672. 
ich kürzlich das Gehirn auf eine andere, etwas genauere Art präpariert, damit dieselben besser sichtbar werden. Wir haben nämlich die weichere, grau getönte Rindensubstanz mit der Messerspitze überall abgekratzt und die weiße, härtere Substanz übriggelassen. Mit diesem Verfahren kann man an verschiedenen Stellen des Gehirns und des verlängerten Marks weiße Bündel zur Anschauung bringen, die wie einzelne Nerven untereinander und mit andern weißen Markgebilden kunstvoll zusammenhängen. Wenn also diese anatomische Präparation besonders die Nervenstränge, die der geheimnisvollen tierischen Bewegung dienen, sehr klar hervorhebt, so ist es angebracht, die eine oder andere Abbildung eines der grauen Hüllschicht beraubten und herausgenommenen Gehirns zu geben ... So haben wir ein herausgenommenes und nach unserer Methode behandeltes Gehirn zwischen den runden Vorwölbungen der 〈Hoden〉 und der 〈Gesäßbacken〉 so durchschnitten, daß sie ohne Beschädigung mit den beiden Hälften der Zirbeldrüse auseinandergeklappt den Hohlraum unter sich zeigen. Dann wird klar, daß die beiden als 〈Testes〉 bezeichneten Vorwölbungen zur weißen Substanz des verlängerten Marks gehören und mit dem Stamm des Kleinhirns zusammenhängen. Sie sind gegen das Großhirn gerichtet und stehen mit ihm in Verbindung» (Seite 24, Kapitel 1). "Commercium inter cerebrum \& cerebellum habeantur» - «Sie mögen Groß- und Kleinhirn miteinander verbinden.» Auf Seite 25 sagt er ferner: «quia plane constat motuum ac sensuum interiorum, aeque ac exteriorum munia, spirituum animalium intra certas, ac distinctas orbitas, seu quasi tubos perexiguos ordinatorum, ope peragi» - «Es ist klar, daß die Spiritus animales der Bewegungen sowie der innern und äußern Sinne in bestimmten wohl von einander zu unterscheidenden Bahnen, die wie kleinste Röhrchen angeordnet sind, geleitet werden.» Die hohlen Nervenröhrchen erinnern an «De Homine» von Descartes (1664), postulieren aber gleichzeitig eine Bahnenlehre. Die paarige Anordnung der Hirnteile erklärt Willis dadurch, daß sie über Verbindungen einander gegenseitig vertreten können: «itu ut si pars una in vitio fuerit, defectus ejus ab altera integra compensetur» - «damit wenn eine Hälfte nicht mehr funktioniert, ihr Ausfall durch die andere voll ersetzt werde.» $\mathrm{Zu}$ beachten ist auch die Legende zu Tafel 5 (Abb.3), wo Willis sagt: «Exhibet Figuram cerebri ovini evoluti, \& quibusdam in locis derasi, \& velut excarnati, ut tractus medullares conspiciantur» - «Sie zeigt ein herausgenommenes Schafhirn, das an einigen Stellen abgeschabt und wie anpräpariert ist, damit es die Markstränge zeige» (Seite 26). Auch in Tafel 6, 7 und 8 (Abb.4) stoßen wir auf die Worte «(d)erasus» oder «excarnatus» zur 


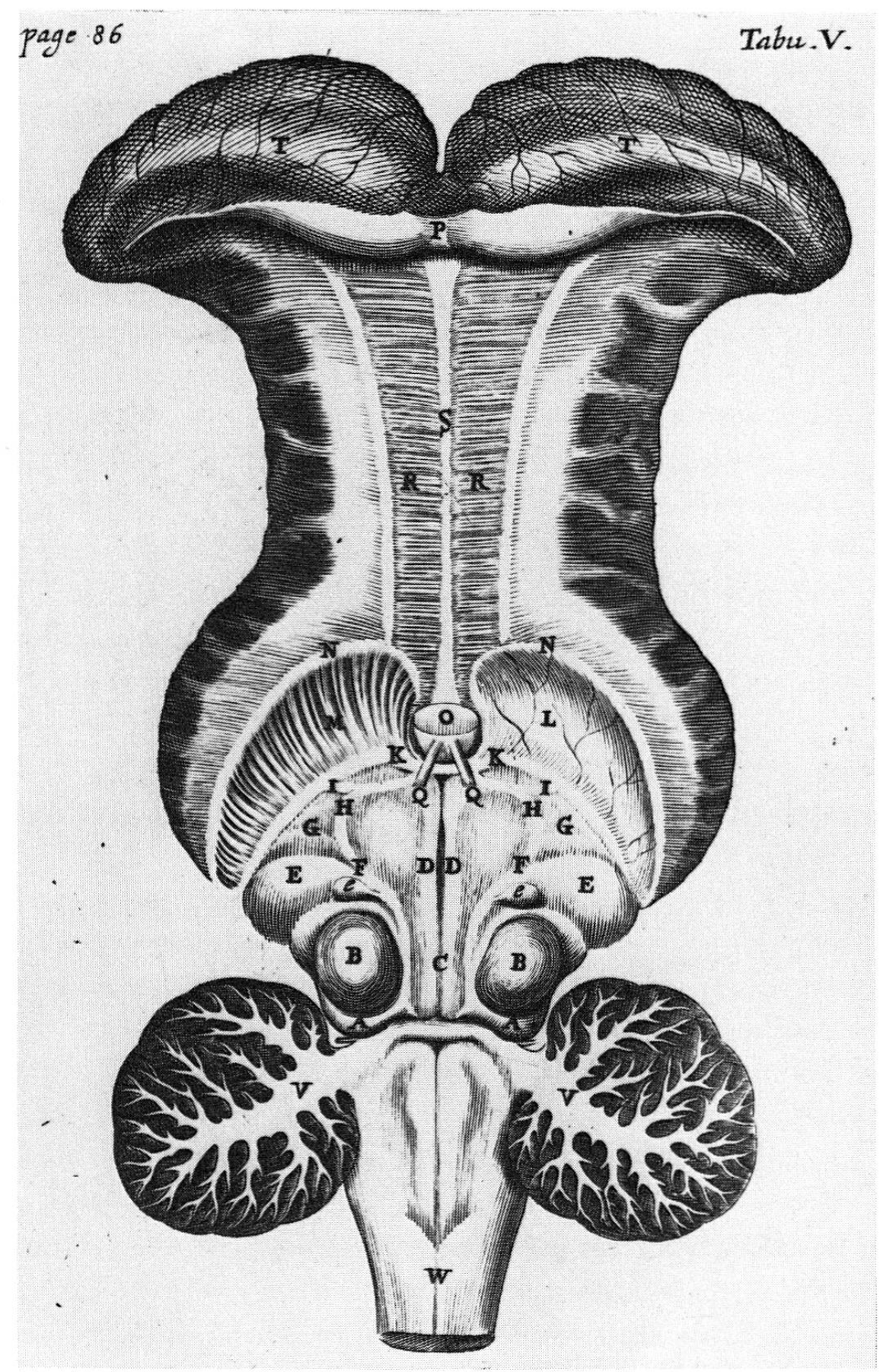

Abb.3. Tabula quinta in «De Anima Brutorum»: «Exhibet Figuram cerebri ovini evoluti, \& quibusdam in locis derasi, \& velut excarnati, ut tractus medullares conspiciantur» - «Sie zeigt ein herausgenommenes Schafhirn, das an einigen Stellen abgeschabt und wie anpräpariert ist, damit es die Markstränge zeige» 
Bezeichnung der neuen Präparationsmethode. Unter «Medulla oblongata» versteht Willis ein Y-förmiges Markgebilde, das aus beiden Großhirnhemisphären entspringt und sich zu einem unpaaren Stamm vereinigt. Für ihn gehören die «Crura cerebri» zur «Medulla oblongata». Die innere Kapsel beginnt dort, wo das «Corpus callosum» im weitern Sinn endigt. Den Tieren schrieb Willis eine materielle «Anima corporea» zu. Sie unterscheidet sich von der «Anima rationalis» des Menschen dadurch, daß sie gleichzeitig mit dem Leben schwindet. Auf Seite 38 formuliert er das: «Cum itaque in

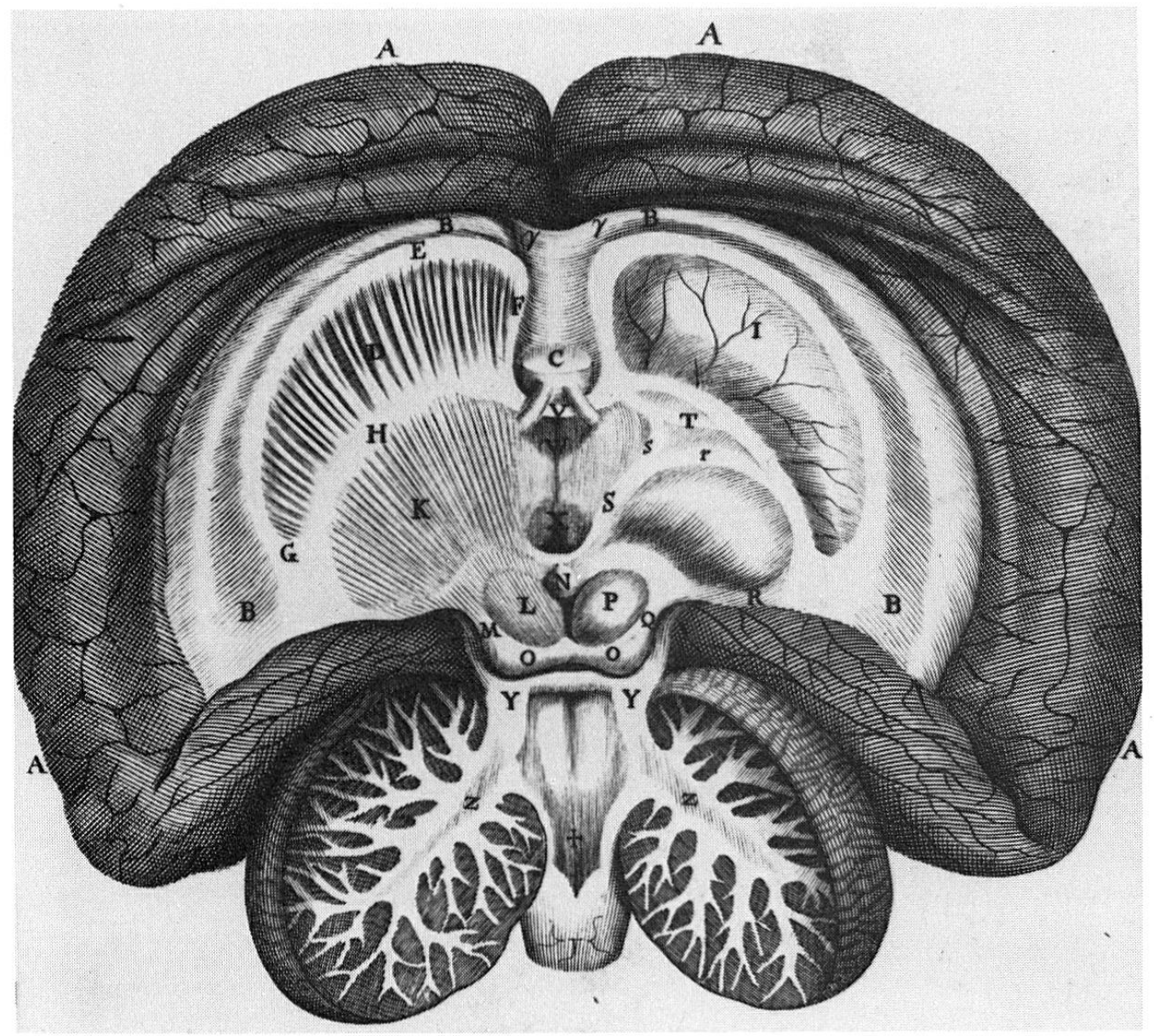

Abb.4. Tabula octava in «De Anima Brutorum»: «Continet novam Cerebri Humani Anatomen, ubi sectione per falcem, corpus callosum $\&$ fornicem factâ, eorumque partibus diductis $\&$ sepositis, corpora striata, nec non prominentiae opticae $\&$ orbiculares, unius lateris erasa $\&$ alterius integra \& plana exhibentur» - «Sie zeigt die neue Präparationsweise des menschlichen Gehirns, bei der nach Durchschneiden von Sichel, Balken und Gewölbe deren Anteile auseinandergezogen und zurückgeklappt wurden, und läßt die Streifenkörper, die Sehhügel und die Vier-Hügel auf der einen Seite abgeschabt, auf der andern Seite ganz und vollständig erkennen» 


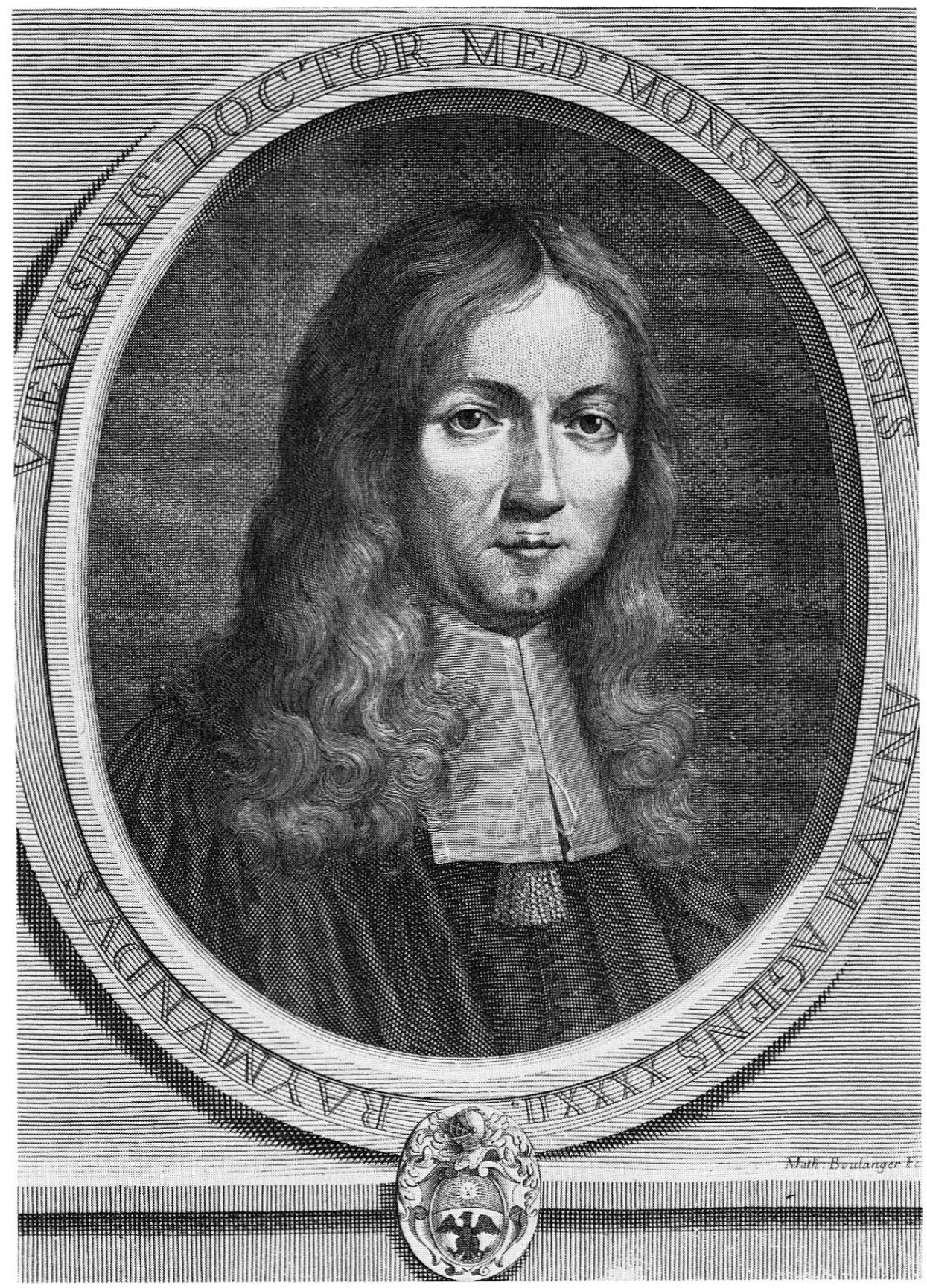

Abb. 5. Porträtstich des Raymund de Vieussens im Alter von 42 Jahren. Gestochen von Math. Boulanger. Vorsatzblatt der «Neurographia universalis», Editio altera, Lyon 1685

homine, praeter animam corpoream, qualem cum brutis communem habet, alia superioris mere spiritualis vestigia, plane detegimus ... - «Neben der Körperseele, die der Mensch mit dem Tiere teilt, haben wir klar die Spuren einer Geistseele gefunden.» 
Raymond de Vieussens (Abb.5)

Leben und Bildungsgang (A. Portal, 1770)

De Vieussens wurde als Sohn eines verarmten Landedelmannes, der bei der Belagerung von Barcelona ums Leben gekommen war, 1641 in Vieussens (Rouergue) geboren. Da die Familie mittellos war, wandte sich der junge Raymond der Medizin zu. Er studierte in Rhodez die Artes liberales, anschließend an der Universität Montpellier Medizin, wo er auch sein Doktordiplom erwarb. Die Praxis übte er als überzeugter Iatrochemiker aus. 1684 wurde er zum Arzt am Spital St. Eloy in Montpellier gewählt. Neben der ärztlichen Praxis interessierte er sich besonders für die Neuroanatomie. Nach dem Dictionnaire des Prosper Marchand soll der junge Vieussens als Vorbereitung auf sein bekanntes Buch, das 1684 in Lyon erschien: «Neurographia universalis, hoc est, omnium corporis humani nervorum simul ac cerebri, medullaeque descriptio anatomica», 560 Autopsien gemacht haben. Aufgrund dieser Veröffentlichung wurde er in die Académie Royale des Sciences in Paris aufgenommen. 1685 ernannte ihn die «Royal Society» in London zu ihrem Mitglied. Vieussens war nie Professor der medizinischen Fakultät von Montpellier. Trotzdem nennt ihn Morgagni in den «Adversaria» (2. Teil) «Montspeliensis Academiae decus et lumen» «Zierde und Leuchte der Universität Montpellier.» 1888 ließ ihn der Marquis de Ward nach Paris rufen. Ludwig XIV wendete ihm eine Jahrespension von 1000 Livres zu. Auch wurde er Leibarzt der Mademoiselle de Montpensier. Seine Ernennung zum «Conseiller d'Etat» erfolgte 1707. Nach dem Tode seiner Gönnerin zog sich De Vieussens nach Montpellier zurück, wo er 1715 starb.

\section{Die «Neurographia universalis» von 1684}

Sie erschien erstmals in Lyon und ist dem Kardinal de Bonsi, Erzbischof von Narbonne, gewidmet. Schon ein Jahr später kam eine «Editio altera» heraus. 1690 erfolgte eine Ausgabe in Ulm und in Frankfurt, 1716 erneut in Lyon und 1775 in Toulouse. Das Werk ist in drei Abschnitte gegliedert. Der erste handelt vom Gehirn, vom Kleinhirn und vom verlängerten Mark, der zweite vom Rückenmark und der dritte vom peripheren Nervensystem. Nach Portal (1770) war Vieussens ein großer Bewunderer der «Cerebri Anatome» des Willis, den er allerdings nirgends mit Namen anführt. 
Vieussens beschreibt die Faserstrukturen der Dura und die Sinus durae matris. Portal (1770) wunderte sich, daß die sehr gute Beschreibung von Lower nicht namentlich erwähnt wird. Dann folgt die Anatomie der Pia mater, in welcher er keine Drüsen finden konnte. Die Beschreibung der Arterien des Großhirns, des Kleinhirns, des verlängerten Markes und des Rückenmarkes ist weniger ausführlich als bei Willis und Lower. Am Gehirn unterschied er graue Rinde und weißes Mark. Mit Malpighi hielt er die Rinde für drüsenartig, während er in der weißen Substanz die Ausführgänge der Rindendrüsen zu sehen glaubte. An jeder Hemisphäre unterschied er 4 Lappen. Die Benennung der Hauptmasse der weißen Substanz als «Centrum semiovale» geht auf Vieussens zurück. Er kannte das Cavum septi pellucidi und die Kommissuren. Er beschreibt genau die Kleinhirnwindungen. Dem Mesencephalondach sollte nach Vieussens eine Klappenfunktion zukommen (Valvula Vieusseni). Gut gelungen ist die Beschreibung der Medulla oblongata, wo er die Pyramiden und die Oliven erwähnte. Im zweiten Abschnitt, der weniger umfangreich als der erste ist, schilderte er die Hüllen und die Form des Rückenmarkes. Bei dessen äußerer Gestalt wies er erstmals auf die Hals- und die Lendenanschwellung hin. Am bedeutendsten ist der dritte Hauptteil, der die besondere Leistung von Vieussens darstellt, die Beschreibung des peripheren Nervensystems. Zunächst handelte er, indem er Willis folgte, von den Nerven, welche aus der Medulla oblongata entspringen. In den Einzelheiten ging er wesentlich über Willis hinaus. Bei den Rückenmarksnerven fällt die Sorgfalt bei der Beschreibung der Plexen auf. Die Schilderung des Sympathicus und der autonomen Geflechte ist sehr gut. Auf Seite 193 seines Werkes beschrieb er die «Ansa Vieusseni»: «Utram que arteriam axillarem nervis velut loris circumligasse videtur» - «Beide Axillararterien werden von Nerven umschnürt wie von Zügeln.» Er glaubte, daß diese Nervenschlingen mechanisch auf die Arterien wirkten. Vorzüglich ist auch die Angabe über den N. vertebralis.

\section{Vieussens und die Präparation der weißen Substanz}

Alle Angaben über die weiße Substanz stehen in Kapitel X des 1. Buches, das den Titel trägt «De distinctis duabus cerebri substantiis» - «Über die zwei verschiedenen Substanzen des Gehirns.» Auf Seite $54^{2}$ beschreibt Vieussens

2 Meine Seitenangaben entsprechen der «Neurographia universalis», Editio altera, Lyon 1685. 
diese beiden Substanzen genauer: «Cerebrum binis a se invicem discrepantibus substantiis, cinerea nempe, \& albâ constat; cinerea autem seu in statu naturali, seu postquam in aqua, vel oleo excocta fuit, spectetur, albâ multò mollior existit: eamque, dum microscopij interpositu, accuratè inspicitur, innumeris è globulis simul coagmentatis, ovalem figuram quadantenùs aemulantibus, constructam esse percipitur» ...- «Das Gehirn besteht aus zwei verschiedenen Substanzen, nämlich einer grauen und einer weißen. Die graue Substanz betrachtet man entweder in ihrem natürlichen Zustand oder nach Kochen in Wasser oder Öl. Sie ist viel weicher als die weiße. Betrachtet man sie genau mit Hilfe des Mikroskopes, so besteht sie aus zahllosen aneinandergefügten Kügelchen von ungefähr ovalärer Form.» Vieussens übernimmt hier die Vorstellung Malpighis, die offensichtlich auf Artefakten beruhte. Er fährt dann weiter: «Praeterea ubi cerebrum in oleo igne lento excocto (quod primus nos docuit Amicus noster Clarissimus Baylé, Doctor Medicus meritissimus, \& in celeberrima omnigenae disciplinae Academiâ Tolosanâ, Artium Professor eruditissimus) illud indurescit» ... - «Wird das Gehirn auf kleinem Feuer in Öl gekocht (wie das unser berühmter Freund Baylé, ein sehr verdienter Arzt und ein sehr gelehrter Philosophieprofessor an der in allen Disziplinen sehr bekannten Universität Toulouse, uns gezeigt hat), so wird es gehärtet» ... - Auf Seite 57 lesen wir: «Cinerea, quam modo descripsimus, substantia, vel exteriorem occupat ambitum cerebri, \& cerebelli ...\& \& haec ubi praedicti visceris exteriora occupat, modò corticis, modò corticalis, modò cinereae, aut glandulosae exterioris substantiae nomine donatur: ubi verò visceris ejusdem interioribus inest, cinereae solùm, aut glandulosae substantiae nomen retinet. Alba cerebri substantia, quam passim substantiam medullarem, imò \& aliquando medullam nominabimus, innumeris è fibrillis simul connexis, ac veluti plures in fasciculos distinctis conflatur ... quod aperte patet dum haec in oleo excoquitur; illà namque tunc temporis innumeris è fibrillis simul connexis ipsam conflatam esse modò diximus, facile dividi potest» - «Die graue Substanz, die wir eben beschrieben haben, nimmt die Oberfläche des Gehirns und des Kleinhirns ein ... Und dort, wo sie die Oberfläche des Organs einnimmt, nennt man sie Rinde, Rindensubstanz, graue Substanz oder äußere Drüsensubstanz. Wo sie aber im Innern liegt, erhält sie nur die Bezeichnung graue Substanz oder Drüsensubstanz. Die weiße Substanz des Gehirns, die wir zuweilen auch als Marksubstanz und gelegentlich auch als Mark bezeichnen, besteht aus zahllosen Fäserchen, die miteinander zusammenhängen, oder aber aus dickeren Faserbündeln, die aus Fäserchen aufgebaut sind. Das tritt deutlich 
hervor, wenn die weiße Substanz in Öl gekocht wird. Dann kann sie leicht in zahllose Fäserchen, wie oben gesagt, zerteilt werden.» Auf die Methode des Abschabens der grauen Substanz nehmen nur die Legenden der Figuren Bezug. So heißt es bei Figur 9 «Cerebrum humanum per superiora dissectum, \& à cinerea substantia exteriore segegratum exhibet» - «Sie zeigt ein von oben präpariertes menschliches Gehirn mit äußerlich abpräparierter grauer Substanz.» Ferner bei Tafel 16 (Abb.6): «Cerebri humani, cerebello, \& vasis omnibus exemptis, a tota ferè cinerea substantia exteriore liberati basim

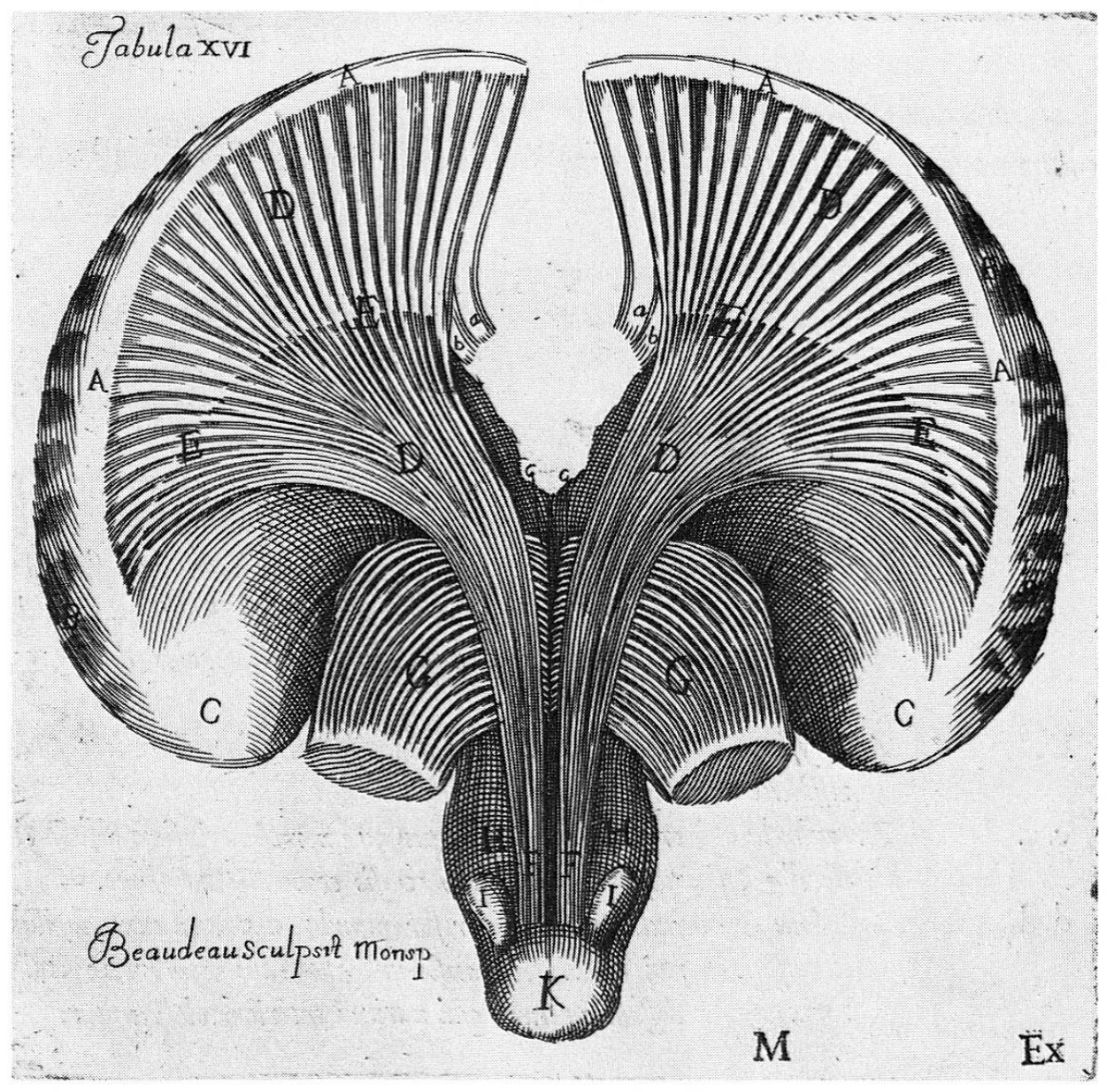

Abb.6. Tafel 16 aus Raymundus de Vieussens «Neurographia universalis», Editio altera, Lyon 1685. Präparation der weißen Faserung, ausgehend von der Hirnrinde durch das «Centrum semiovale Vieusseni», die innere Kapsel und die beiden Pyramiden. Darstellung der weißen Faserung zwischen Brücke und Kleinhirn. Die Abbildung, die von Beaudeau gestochen wurde, gibt eine gute Vorstellung von der Leistungsfähigkeit der Abrasionsmethode nach Vieussens 
paulò profundius, quam in Tabula praecedenti derasam exhibet»-«Sie zeigt eine menschliche Hirnbasis nach Wegnahme des Kleinhirns und aller Gefäße. Fast die ganze graue Substanz ist äußerlich abgekratzt, und zwar etwas tiefer als in der vorhergehenden Tafel.» Figur 138 und 139 sollen die Struktur der weißen Substanz verdeutlichen, erreichen das gesetzte Ziel aber nur unvollkommen und tragen verhältnismäßig wenig zum bessern Verständnis des Textes bei. Nach Portal (1770) bezahlte Vieussens dem Kupferstecher Beaudeau von Montpellier 24 Livres pro Tag.

In seiner «Neurographia Universalis» von 1684 macht Vieussens ausgiebigen Gebrauch vom Abkratzen der grauen Rinde. Seine technischen Angaben sind viel weniger genau als bei Willis' «De Anima Brutorum» von 1672. Ohne jeden Zweifel kommt Willis die Priorität bei der Präparation der weißen Substanz zu. Vieussens erwähnt ihn jedoch nicht. Möglicherweise kannte er nur seine «Cerebri Anatome» von 1664, die er nach Portal sehr geschätzt hat. Für die Verfestigung der Hirnsubstanz in kochendem Öl gibt er einen Gewährsmann an, den Toulouser Professor Baylé. Die Methode geht jedoch auf Marcello Malpighi (1665) zurück. Den «Discours» Stensens erwähnt Vieussens nirgends.

\section{Faserpräparate}

Stensen hat in seinem «Discours», den er 1665 hielt, der aber erst 1669 gedruckt wurde, eindrücklich auf die Bedeutung einer Präparation der weißen Faserbündel hingewiesen. Daß er jedoch diese Methode selber zum Präparieren des Gehirns mit Erfolg gebraucht habe, ist nirgends zu belegen. Von den beiden Hirnanatomen, die die «Abrasionsmethode» geübt haben, kommt Willis (1672) gegenüber Vieussens (1684) die Priorität zu. Ob Willis Stensens «Discours» gekannt hat, wissen wir nicht, ebensowenig, ob Vieussens den «Discours» Stensens oder «De Anima Brutorum» des Willis gelesen hat. Die «Abrasionsmethode» ist zweifelsohne der früheste Versuch einer präparatorischen Darstellung der weißen Substanz, wenn man sie auch nicht als eine eigentliche «Abfaserungsmethode» betrachten kann. Dazu brauchte es zusätzliche neue Methoden, die Formolfixation, die 1893 von Blum eingeführt worden ist, und das Durchfrieren der fixierten Gehirne, was erstmals Gennari (1782) in größerem Ausmaß geübt hat. Das Verdienst, diese beiden zusätzlichen Methoden in den Dienst der Abfaserung der weißen Substanz gestellt zu haben, kommt Klingler (1935) zu. Die Faserpräparate 
(Ludwig und Klingler, 1938 und 1956) zeigen eine bis dahin nie erreichte Schönheit und Klarheit. So hat es fast 300 Jahre gedauert, bis die von Stensen zuerst geäußerte Idee volle Wirklichkeit geworden ist.

\section{Schrifttum}

Bartholin, C. Thom. Filius: Administrationum anatomicarum Specimen, in quo selectiora quaedam operi majori praemittuntur. Francofurti 1679.

Bierbaum, M. $\dagger$ und Faller, A.: Niels Stensen. Anatom, Geologe und Bischof. Münster (Westfalen) 1979.

Blum, F.: Der Formaldehyd als Härtungsmittel. Zsch. wiss. Mikroskopie 10 (1893) 314-15.

Burdach, K.F.: Vom Bau und Leben des Gehirns. Bd.3. Leipzig 1822.

Clarke, E. and O'Malley, C.D.: The Human Brain and Spinal Corde. A Historical Study. Berkeley Los Angeles 1968.

Descartes, R.: De Homine. Figuris et Latinitate donatus a Florentio Schuyl, Inclitae Urbis Sylvae Ducis Senatore, \& ibidem Philosophiae Professore. Lugduni Batavorum 1662.

Fanoisius, G.: Nicolai Stenonis de Cerebri Anatome Dissertatio, spectatissimis viris DD societatis apud Dominum Thévenot Parisiis collecta, dicata atque a Gallico exemplari Parisiis edita An. 1669, Latinitate donata, Opera et Studio Guidonis Fanoisii LL. AA.M. et Med. Doctor. Lugduni Batavorum 1671.

Faller, A.: Wertschätzung von Stensens «Discours sur l'anatomie du cerveau» im Verlaufe von drei Jahrhunderten. Bd.35 der Veröffentlichungen der Schweizerischen Gesellschaft für Geschichte der Medizin und der Naturwissenschaften. Aarau/Frankfurt am Main 1981.

Fontana, F.G.F.: Observations sur la structure des nerfs faites à Londres en 1779. In Vol. 2 Traité sur le venin de la vipère. Florence 1781, 187-208.

Gennari, Fr.: De peculiari structura cerebri nonnullisque ejus morbis. Parma 1782.

Isler, H. R.: Thomas Willis. Große Naturforscher, Bd.29. Wissenschaftliche Verlagsgesellschaft, 1965.

Klingler, J.: Erleichterung der makroskopischen Präparation des Gehirns durch den Gefrierprozeß. Schweiz. Archiv Neurol. u. Psych. 36 (1935) 247-256.

Larsen, K. und Scherz, G.: Nicolai Stenonis Opera theologica. Tomus posterior. Hafniae 1947. «Parochorum hoc age seu evidens demonstratio, quod parochus teneatur omnes alias occupationes dimittere et suae intendere perfectioni, ut commissa sibi oves ad statum salutis aeternae ipsis a Christo praeparatum perducat», p. 12.

Ludwig, E. und Klingler, J.: Kerne und Faserbahnen des menschlichen Gehirns. 50 Heliogravüren nach makroskopischen Präparaten der Anatomischen Anstalt in Basel. Nancy 1938.

- Atlas cerebri humani. Basel/New York 1956.

Maar, V.: Nicolai Stenonis Opera philosophica. Vol.2. Copenhagen 1910. Discours sur l'anatomie du cerveau. p.1. Elementorum Myologiae Specimen, seu Musculi Descriptio geometrica. p.61. Canis Carchariae Dissectum Caput. p.113. De Solido intra solidum naturaliter contento Dissertationis Prodromus. p.181. Prooemium Demonstrationum anatomicarum in Theatro Hafniensi Anni 1673. p. 249.

Malpighi, M.: De Cerebro Epistola. Bononiis 1664.

Morgagni, G. B. M.: Adversaria anatomica II. Padua 1717. 
Piccolomini, A.: Anatomicae praelectiones explicantes mirificam corporis humani fabricam. Romae 1586.

Portal, A.: Histoire de l'anatomie et de la chirurgie, contenant l'origine et le progrès de ces Sciences. Tome 4. Paris 1770.

Sprengel, K.: Versuch einer pragmatischen Geschichte der Arzneikunde. 5 Bde. Halle $1800-03$.

Steno, N.: Nicolaus Steno's Lecture on the Anatomy of the Brain. Introduction by Gustav Scherz. Copenhagen 1965.

Vieussens de, R.: Raymundi Vieussens Doctoris monspeliensis Neurographia universalis. Hoc est, omnium corporis humani nervorum, simul \& cerebri, medullaeque spinalis descriptio anatomica; eaque integra et accuarata variis iconibus fideliter \& ad vivum delineatis, aereque incisis illustrata: cum ipsorum actione et usu, physico discursu explicatis. Editio nova. Lugduni 1685.

Willis, Th.: Cerebri Anatome: cui accessit Nervorum Descriptio et Usus. Londini 1664. Pathologiae cerebri et Nervosi Generis Specimen. Londini 1667. De Anima Brutorum quae Hominis Vitalis ac Sensitiva est, Exercitationes duae, Quarum Prior Physiologica etc. Altera Pathologica. Oxonii 1672.

\section{Zusammenfassung}

In seinem «Discours sur l'anatomie du cerveau» (gehalten 1665, gedruckt 1669) hat Nicolaus Stenonis (1638-1668) erstmals auf die große Bedeutung einer Präparation der weißen Faserbündel im Gehirn hingewiesen. Die frühesten Versuche einer solchen Darstellung werden als «Abrasio», «(D) Erasio» oder «Excarnatio» bezeichnet. Von den beiden Neuroanatomen des 17.Jahrhunderts, die solche Präparate abgebildet haben, kommt Thomas Willis (1621-1675) in «De Anima Brutorum» (1672) die Priorität zu gegenüber Raymundus de Vieussens (1661-1716), dessen «Neurographia universalis» 1684 in Lyon erschien und der meist als Erfinder dieser Präparationsmethode gilt.

\section{Summary}

In his «Discours sur l'anatomie du cerveau» (1665, printed in 1669) Nicolaus Stenonis (1638-1686) for the first time stressed the importance of a preparation of the white fascicles in the brain. The earliest attempts of such a representation are there called "Abrasio", "(D) Erasio" or "Excarnatio". Among the two neuroanatomists of 17th century who have produced such preparations Thomas Willis (1621-1675), in his "De Anima Brutorum" (Oxford 1672) deserves priority in relation to Raymundus de Vieussens (1661-1716) whose "Neurographia universalis" (1684) was published in Lyons and who, wrongly, is not seldom considered the inventor of this method of preparation under review.

Prof. Dr. med. Adolf Faller

Bibliothek für Medizingeschichte, 1, rue Gockel, CH-1700 Fribourg 
